\title{
Redundant roles of the phosphatidate phosphatase family in triacylglycerol synthesis in human adipocytes
}

\author{
Ana Temprano ${ }^{1,2}$ - Hiroshi Sembongi ${ }^{3,4} \cdot$ Gil-Soo Han $^{5}$ - David Sebastián ${ }^{6,7,8}$. \\ Jordi Capellades $^{8,9}$ • Cristóbal Moreno ${ }^{1,8}$ • Juan Guardiola ${ }^{10}$ • Martin Wabitsch ${ }^{11}$. \\ Cristóbal Richart $^{1,12}$ - Oscar Yanes ${ }^{8,9,13}$ - Antonio Zorzano ${ }^{6,7,8}$ - George M. Carman ${ }^{5}$. \\ Symeon Siniossoglou ${ }^{3} \cdot$ Merce Miranda $^{1,8}$
}

Received: 22 April 2016 / Accepted: 23 May 2016 / Published online: 25 June 2016

(C) The Author(s) 2016. This article is published with open access at Springerlink.com

\begin{abstract}
Aims/hypothesis In mammals, the evolutionary conserved family of $\mathrm{Mg}^{2+}$-dependent phosphatidate phosphatases (PAP1), involved in phospholipid and triacylglycerol synthesis, consists of lipin-1, lipin-2 and lipin-3. While mutations in the murine Lpin 1 gene cause lipodystrophy and its knockdown in mouse 3T3-L1 cells impairs adipogenesis, deleterious mutations of human LPIN1 do not affect adipose tissue distribution. However, reduced LPIN1 and PAP 1 activity has been described in participants with type 2 diabetes. We aimed to characterise
\end{abstract}

Electronic supplementary material The online version of this article (doi:10.1007/s00125-016-4018-0) contains peer-reviewed but unedited supplementary material, which is available to authorised users.

Symeon Siniossoglou

ss560@cam.ac.uk

$\triangle$ Merce Miranda

mmg@mercemiranda.net

1 Joan XXIII University Hospital, Pere Virgili Health Research Institut (IISPV), Modular Building, C/ Mallafre Guasch, Tarragona 43005, Spain

2 Department of Biochemistry and Molecular Biology, Rovira i Virgili University, Tarragona, Spain

3 Cambridge Institute for Medical Research, University of Cambridge, Wellcome Trust/Medical Research Council Building, Hills Road, Cambridge CB2 0XY, UK

4 Present address: Chesterford Research Park, Little Chesterford, Saffron Walden, UK

5 Department of Food Science and the Rutgers Center for Lipid Research, New Jersey Institute for Food, Nutrition and Health, Rutgers University, New Brunswick, NJ, USA the roles of all lipin family members in human adipose tissue and adipogenesis.

Methods The expression of the lipin family was analysed in adipose tissue in a cross-sectional study. Moreover, the effects of lipin small interfering RNA (siRNA)-mediated depletion on in vitro human adipogenesis were assessed.

Results Adipose tissue gene expression of the lipin family is altered in type 2 diabetes. Depletion of every lipin family member in a human Simpson-Golabi-Behmel syndrome (SGBS) pre-adipocyte cell line, alters expression levels of adipogenic transcription factors and lipid biosynthesis genes
6 Institute for Research in Biomedicine (IRB Barcelona), The Barcelona Institute of Science and Technology, Barcelona, Spain

7 Department of Biochemistry and Molecular Biology, Faculty of Biology, University of Barcelona, Barcelona, Spain

8 Biomedical Research Networking Centre in Diabetes and Associated Metabolic Disorders (CIBERDEM), Instituto de Salud Carlos III, Madrid, Spain http://www.ciberdem.org/

9 Centre for Omic Sciences, Rovira i Virgili University, Reus, Spain

10 Department of Pulmonary, Critical Care and Sleep Medicine, University of Louisville, Louisville, KY, USA

11 Division of Paediatric Endocrinology and Diabetes, Interdisciplinary Obesity Clinic, University Clinic for Child and Adolescent Medicine, University of Ulm, Ulm, Germany

12 GEMMAIR Research Group - Applied Medicine, Department of Medicine and Surgery, Rovira i Virgili University (URV), Tarragona, Spain

13 Department of Electronic Engineering, Rovira i Virgili University, Tarragona, Spain 
in early stages of differentiation. Lipin-1 knockdown alone causes a 95\% depletion of PAP1 activity. Despite the reduced PAP1 activity and alterations in early adipogenesis, lipinsilenced cells differentiate and accumulate neutral lipids. Even combinatorial knockdown of lipins shows mild effects on triacylglycerol accumulation in mature adipocytes.

Conclusions/interpretation Overall, our data support the hypothesis of alternative pathways for triacylglycerol synthesis in human adipocytes under conditions of repressed lipin expression. We propose that induction of alternative lipid phosphate phosphatases, along with the inhibition of lipid hydrolysis, contributes to the maintenance of triacylglycerol content to near normal levels.

Keywords Basic science $\cdot$ Cell lines $\cdot$ Human $\cdot$ Lipid metabolism

$\begin{array}{ll}\text { Abbreviations } \\ \text { DAG } & \text { Diacylglycerol } \\ \text { DNL } & \text { De novo lipogenesis } \\ \text { ER } & \text { Endoplasmic reticulum } \\ \text { FAO } & \text { Fatty acid oxidation } \\ \text { LPP } & \text { Lipid phosphate phosphatases } \\ \text { ORO } & \text { Oil Red O staining } \\ \text { PAP } & \text { Phosphatidate phosphatase } \\ \text { PPAR } & \text { Peroxisome proliferator activated receptor } \\ \text { SGBS } & \text { Simpson-Golabi-Behmel syndrome } \\ \text { siRNA } & \text { Small interfering RNA } \\ \text { SAT } & \text { Subcutaneous adipose tissue } \\ \text { TAG } & \text { Triacylglycerol } \\ \text { VAT } & \text { Visceral adipose tissue }\end{array}$

\section{Introduction}

Triacylglycerols (TAGs) are neutral lipids that act as the major energy storage molecules, repository for fatty acids, and phospholipid precursors [1]. Adipocytes are the specialised cells for neutral lipid storage and one of their important physiological functions is to buffer the toxicity caused by NEFAs. Excessive calorie intake or genetic disorders can lead to lipid deposition in ectopic tissues, impair their function and lead to dyslipidaemia, insulin resistance and type 2 diabetes $[2,3]$.

Lipins are $\mathrm{Mg}^{2+}$-dependent phosphatidate phosphatases (PAP1) with a central role in lipid metabolism, and catalyse the dephosphorylation of phosphatidate to diacylglycerol (DAG), which can be (1) acylated to form TAG, or (2) used in phospholipids synthesis $[4,5]$. A second type of PAP activity is mediated by $\mathrm{Mg}^{2+}$-independent transmembrane lipid phosphate phosphatases (LPPs, also known as PAP2), which are thought to regulate signalling properties of phosphatidate and DAG [6].

Fungi, nematodes and insects express one lipin, whereas mammals express three paralogues called lipin-1, -2 and -3 that exhibit distinct but overlapping expression in many mouse and human tissues [5]. Consistent with their key metabolic role, loss of lipin function disrupts TAG production, membrane organisation and phospholipid synthesis in several model organisms [7]. Interestingly, besides their enzymatic functions, lipins also regulate transcription [8-13]. For instance, lipin-1 transcriptional co-regulation of the peroxisome proliferator activated receptor (PPAR)A/PPAR coactivator $1 \alpha$ axis modulates fatty acid oxidation (FAO) in liver [9].

Lpin1 gene was originally identified as the deficient gene causing lipodystrophy, insulin resistance, peripheral neuropathy and neonatal fatty liver in the fld (also known as Lpin1) mouse model [14]. Loss of lipin-1 in mice blocks adipogenesis at an early stage preceding TAG accumulation, suggesting a distinct role of lipin-1 in differentiation $[15,16]$. Consistently, small interfering RNA (siRNA)-mediated silencing of lipin-1 in mouse 3T3-L1 cells potently inhibits adipogenesis [10, 17]. In contrast, deleterious mutations in the LPIN1 gene in humans, which lead to recurrent rhabdomyolysis in childhood, do not compromise adipose tissue [18]. The basis of this difference between mice and humans is unknown; it has been hypothesised that it is due to compensation by the other two lipins [18]. Nevertheless, genetic variation in LPIN1 and LPIN2, and reduced LPIN1 expression levels and PAP1 activity in human adipose tissue have been associated with type 2 diabetes [19-25], which suggests a loss of their protective role against lipotoxicity.

While most of the studies have been performed on mice, very little is known on the roles of lipin-1 in human adipocyte physiology, while there is virtually no information on lipin-2 and -3 . This prompted us to investigate the functions of the three lipins in type 2 diabetes and in human adipocytes by studying the effects of loss-of-function.

\section{Methods}

Reagents Unless otherwise stated, all reagents were supplied by Sigma-Aldrich Corporation (St. Louis, MO, USA). Human insulin was purchased from NovoNordisk (Bagsværd, Denmark); rosiglitazone from Cayman Chemical (Ann Arbor, MI, USA); and cell culture media from Gibco (Thermo Fisher Scientific, Waltham, MA, USA).

Human adipose tissue biopsy collection The cross-sectional study has been previously described [26, 27]. We used a cohort of 71 participants for the gene expression analysis, and of 28 males for protein analysis. They were grouped as: 
(1) normoweight (BMI between 18.50 and 24.99), (2) obesity (BMI $\geq 25$ ) and (3) obesity with type 2 diabetes (hereafter type 2 diabetes). Group characteristics are described in electronic supplementary material (ESM) Table $1 \mathrm{~A}$ and 1B, respectively. Samples were obtained at the Joan XXIII University Hospital (Tarragona, Spain). VAT and SAT samples (gene expression) and SAT samples (protein expression) were obtained during abdominal elective surgical procedures for benign pathologies (cholecystectomy or surgery for abdominal hernia). The Ethics Committee approved the study and informed consent was obtained from all participants. Participants had no systemic disease other than obesity [28] or type 2 diabetes [29]. See ESM Methods for further details.

Cell culture and differentiation Simpson-Golabi-Behmel syndrome (SGBS) cells, a well-established system for studies of human adipocyte biology [30,31], were differentiated as described [30], except that $1 \mathrm{nmol} / 1$ of insulin, $0.1 \mu \mathrm{mol} / 1$ cortisol, $2 \mu \mathrm{mol} / \mathrm{l}$ rosiglitazone and $25 \mathrm{nmol} / 1$ dexamethasone were used. See ESM Methods for detailed information.

Adipose-derived stem cells were isolated from adipose tissue ( $n=3$ female donors; age (years) $37.4 \pm 6.4$, BMI $\left.\left(\mathrm{kg} / \mathrm{m}^{2}\right) 25.9 \pm 3.0\right)$ from patients undergoing elective liposuction surgery. See ESM Methods for the isolation, proliferation and adipogenic procedure details.

Quantification of expression levels Protein content in cell lysates (lysis buffer: $50 \mathrm{mmol} / \mathrm{l}$ HEPES pH 7.4, $150 \mathrm{mmol} / \mathrm{l}$ $\mathrm{NaCl}, 4 \mathrm{mmol} / 1 \mathrm{MgCl}_{2}, 1 \%$ Triton X-100 and protease inhibitors) was quantified by using the bicinchoninic acid assay (Pierce, Rockford, IL, USA). See ESM for western blot procedures. Antibodies against lipin family members were previously described $[17,32]$. Quantitative PCR was performed as previously described [27], and expressed relative to cyclophilin A and to control. See ESM for detailed information and ESM Table 2A and 2B for commercial reagents.

Cell Fractionation For cell fractionation, SGBS cells were grown and differentiated until day 10. Subcellular fractions were obtained by using hypotonic lysis followed by high salt extraction of nuclei. See ESM Methods for further details.

Gene silencing Transfections of SGBS cells with siRNA oligonucleotides were carried out by using Lipofectamine RNAiMAX transfection reagent (Thermo Fisher Scientific). Pre-adipocyte knockdowns were performed as a two-shots transfection of a mix of two siRNA duplexes per gene (12.5 nmol/1 of each duplex) (see ESM Table 2C): reverse transfection at the start of the experiment and forward transfection the day before confluence. The non-targeting control concentration depended on total siRNA amount of the single and multiple knockdowns.
Neutral lipid accumulation and metabolism Data were obtained from differentiated SGBS cells and normalised by protein content. For TAG analysis, cells were processed as previously described [32], except that the supernatant fraction was analysed with the Serum Triglyceride Determination kit (Sigma). Glycerol release to cell culture media was quantified by using the Free Glycerol Determination Kit (Sigma). Fatty acid and glucose incorporation into TAGs, and FAO analysis were performed as previously described [33], with slight modifications (see ESM Methods for details).

Enzyme assay Cell lysates (lysis buffer: $50 \mathrm{mmol} / \mathrm{l}$ Tris- $\mathrm{HCl}$ $\mathrm{pH} 7.5,0.25 \mathrm{~mol} / \mathrm{l}$ sucrose, $10 \mathrm{mmol} / \mathrm{l}$ 2-mercaptoethanol, protease inhibitors) were subjected to centrifugation at 1000 $g$ for $10 \mathrm{~min}$ at $4^{\circ} \mathrm{C}$ to remove cell debris. Protein concentration was determined by the method of Bradford [34] using BSA as a reference protein. Preparation of the substrate and measure of PAP activity was as previously described [35-37]. See ESM Methods for details. The $\mathrm{Mg}^{2+}$-independent LPP activity was measured in the same reaction mixture except that $2 \mathrm{mmol} / \mathrm{l}$ EDTA was substituted for $0.5 \mathrm{mmol} / 1 \mathrm{MgCl}_{2}$. The $\mathrm{Mg}^{2+}$-dependent PAP1 activity was determined by subtraction of LPPs activity from PAP activity. A unit of PAP activity (expressed as units/mg protein) was defined as the amount of enzyme catalysing the formation of $1 \mathrm{nmol}$ of product/min.

Metabolomic analysis SGBS cells were grown, transfected with siRNA as explained above, and differentiated to day 4 . In brief, lipids were extracted from lyophilised samples by using dichloromethane/methanol and water. The organic phase (lipidic) was collected, dried under a stream of nitrogen, and resuspended in acetonitrile/isopropanol/water for untargeted LC-MS analysis. Differentially regulated lipids ( $p$ value $<0.05$ and fold $>2$ ) were retained for compound identification by MS/MS analyses. See ESM Methods for detailed procedures.

Statistical analysis Statistical analysis was performed by using the SPSS software version 15 (Chicago, IL, USA). ANOVA, Kruskal-Wallis, Pearson $\chi^{2}$, Spearman correlation and Linear Stepwise Regression tests were performed for the human cohort analysis, and the General Linear Model Univariate test for in vitro experiments. Statistical power in the cohort analysis was $\geq 80 \%$. The level of significance was set at $\alpha=0.05$.

\section{Results}

Adipose tissue expression levels of the lipin family is altered in type 2 diabetes To investigate the roles of lipin paralogues in adipose tissue, we started by examining their gene expression in paired abdominal subcutaneous (SAT) and visceral (VAT) adipose tissue biopsies. As shown in 

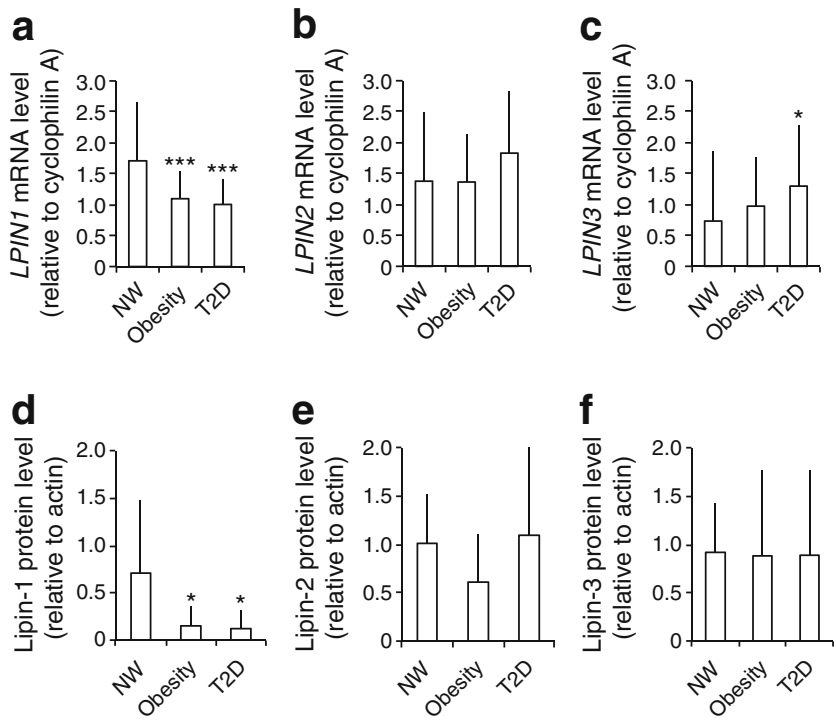

Fig. 1 Altered expression of the lipin family in type 2 diabetes adipose tissue. Participants were grouped by BMI and type 2 diabetes (T2D). $(\mathbf{a}-\mathbf{c})$ mRNA expression relative to cyclophilin $\mathrm{A}$ and to a calibrator that consisted of a mix of mRNA samples $(n=17,43$ and 11 samples for the normoweight [NW], obesity and T2D groups, respectively), and (d-f) protein expression normalised to actin $(n=9,10$ and 9) were quantified in human abdominal subcutaneous adipose tissue. Data represent mean $\pm \mathrm{SD}$; ${ }^{*} p<0.05,{ }^{* * *} p<0.001$ vs normoweight; ANOVA and Kruskal-Wallis tests

Fig. 1a-c and ESM Fig. 1a-c, LPIN1 expression was reduced in both SAT $(p<0.001)$ and VAT $(p=0.021)$ in the obesity and type 2 diabetes groups compared with normoweight. LPIN2 expression was similar among groups. In contrast, SAT LPIN3 expression was significantly increased in the type 2 diabetes $(p=0.018)$. At the protein level, SAT showed only lipin-1 was downregulated in obesity and in type 2 diabetes compared with normoweight $(p=0.034)$ (Fig. 1d-f).

Correlation analysis showed a negative association of LPIN1 expression levels with BMI, HOMA-IR and plasma TAG levels. In contrast, expression of LPIN3 positively correlates with fasting glucose (SAT) and NEFA (VAT) (Table 1). Regression analysis was performed, assessing age and sex as confusing and interacting variables, and showed that (1) SAT LPIN1 expression depends negatively on HOMA-IR $(R=0.466, p=0.005$, excluded variables: BMI, TAG, age and sex; $\left.L P I N 1_{\mathrm{SAT}}=0.37 \times \log _{10} \mathrm{HOMA}+0.984\right)$, and (2) SAT LPIN3 expression depends positively on plasma glucose levels $(R=0.414, p=0.002$, excluded variables: age and sex; $\left.L P I N 3_{\text {SAT }}=1 \times 10^{\left[1.202 \times \log _{10} \text { Glucose- } 0.961\right]}\right)$.

The observed changes of the lipin family expression in type 2 diabetes participants may account for the altered PAP1 activity in adipocytes from these patients. Moreover, LPIN3 expression is associated with fasting glucose levels. Alterations in lipin-3 protein levels in SAT of participants with type 2 diabetes may be masked by its presence also in the stromal vascular fraction (data not shown).

\section{The three lipin family members have a role in early human} adipogenesis Next, we examined the expression of lipins during adipogenesis. Lipin-1 was induced, and lipin-2 and lipin-3 levels were present along SGBS adipogenesis with slight variation (Fig. 2a, b). Thus, SGBS adipocytes, similar to differentiated adipose-derived stem cells (ESM Fig. 1d), express the three lipin paralogues. Finally, under baseline conditions, the three lipins partitioned between the cytosolic, intranuclear and membrane-bound forms in SGBS adipocytes (ESM Fig. 1e).

To address the effects of decreased PAP1 activity on adipogenesis, we depleted each lipin member prior to the induction of adipogenesis in SGBS pre-adipocytes (by using siRNA; see Methods and Fig. 2c). Cells were analysed at day 4 after differentiation to assess adipogenic early events. Protein expression analysis was used to confirm knockdowns and evaluate possible compensatory mechanisms among lipins. Lipin-1 depleted cells responded by compensatory upregulation of lipin-2 protein (Fig. 2d and ESM Fig. 1f), but not LPIN2 transcript levels (data not shown). Conversely, single lipin-2 and lipin-3 knockdowns led to lower protein levels of the other family members (Fig. 2d).
Table 1 Correlation analysis in abdominal SAT and VAT adipose tissue biopsies from 71 participants

\begin{tabular}{|c|c|c|c|c|c|c|}
\hline \multirow[t]{2}{*}{ Variable } & \multicolumn{3}{|l|}{ SAT } & \multicolumn{3}{|l|}{ VAT } \\
\hline & LPIN1 & LPIN2 & LPIN3 & LPIN1 & LPIN2 & LPIN3 \\
\hline BMI & $-0.349 * *$ & -0.021 & 0.220 & $-0.283^{\dagger}$ & -0.091 & 0.034 \\
\hline HOMA-IR & $-0.471 * *$ & -0.104 & -0.006 & $-0.329^{*}$ & -0.139 & -0.006 \\
\hline Glucose & $-0.349 * *$ & $0.253^{\dagger}$ & $0.357^{* *}$ & -0.119 & 0.189 & $0.298^{\dagger}$ \\
\hline Insulin & $-0.522 * * *$ & -0.169 & -0.039 & $-0.340 * *$ & -0.179 & 0.017 \\
\hline Triacylglycerol & $-0.319^{* *}$ & -0.018 & 0.072 & -0.202 & 0.131 & 0.238 \\
\hline NEFA & -0.095 & 0.129 & 0.128 & -0.029 & 0.125 & $0.336^{* *}$ \\
\hline Glycerol & -0.188 & -0.105 & 0.144 & -0.113 & 0.097 & $0.274^{\dagger}$ \\
\hline
\end{tabular}

Spearman coefficient of the correlation analysis is shown. ${ }^{*} p<0.05$, ** $p<0.01,{ }^{* * *} p<0.001$

Where statistical power is lower than $80 \%:^{\dagger} p<0.05$ 
Fig. 2 Single knockdowns of lipins in SGBS pre-adipocytes. SGBS pre-adipocytes were induced to differentiate, and mRNA (a) and protein (b) levels were analysed during adipogenesis at the given time points $(n=3)$. Data represent mean \pm SD of fold induction over day 0. (c) Knockdowns of single lipin members were performed in pre-adipocytes, adipogenesis was induced (day 0 ) and cells were collected at day 4. (d) Lipin protein levels $(n=7)$, (e) PAP1 activity (control: 23.10

$\left.\pm 5.12 \mathrm{nmol} \mathrm{min}^{-1} \mathrm{mg}^{-1}\right)(n=3)$, (f) fatty acid (FA) and glucose incorporation into TAGs (control, fatty acids: 220.8

\pm 185.7 arbitrary units TAG/ $\mu \mathrm{g}$ protein, glucose: 3.47

\pm 6.30 arbitrary units TAG/ $\mu \mathrm{g}$ protein) $(n=3)$, and mRNA levels of (g) early adipogenic

transcription factors $(n=3)$, and (h) lipogenic genes $(n=3)$ were analysed. Gene expression is expressed relative to cyclophilin A and to non-targeting control. Protein expression is normalised to actin levels. Data represent mean \pm SD of fold increase over non-targeting controls (set as 1 ). ${ }^{*} p<0.05,{ }^{* *} p<0.01$, $* * * p<0.001$, General Lineal

Model Univariate test. $(\mathbf{a}, \mathbf{b})$

White squares, LPIN1; grey squares, $L P I N 2$; black squares, LPIN3; (d-h) white bars, LPIN1 knockdown; grey bars, LPIN2 knockdown; black bars, LPIN3 knockdown a

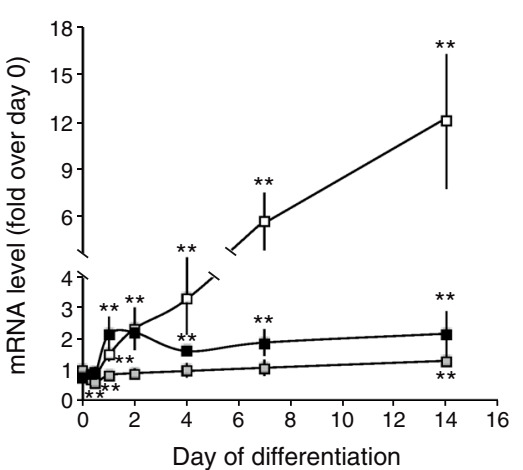

C

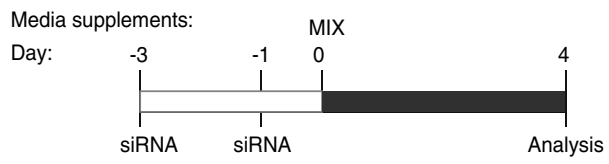

d
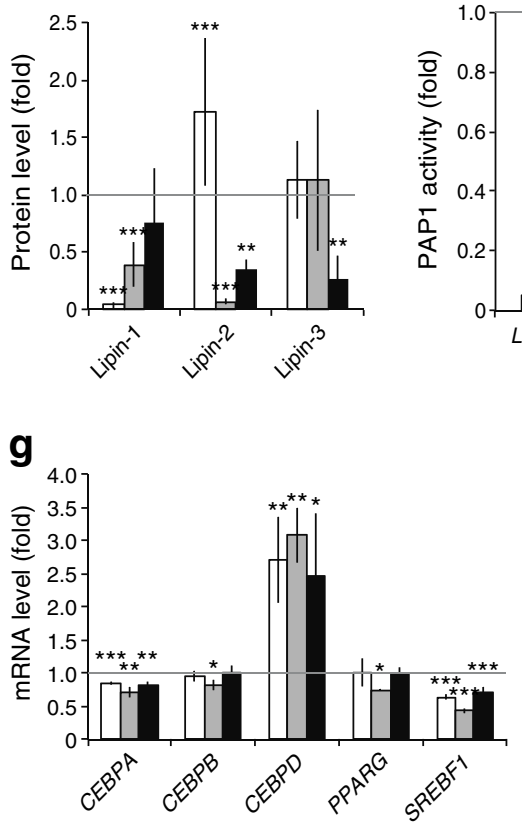

b

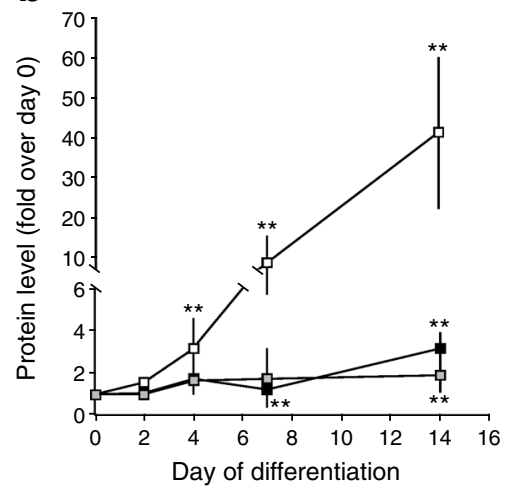

e

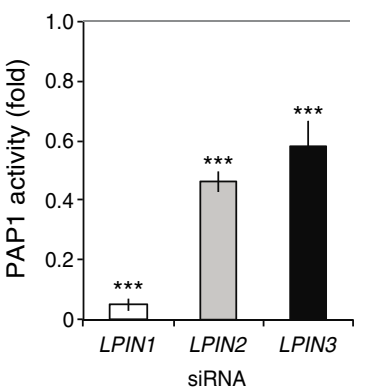

f

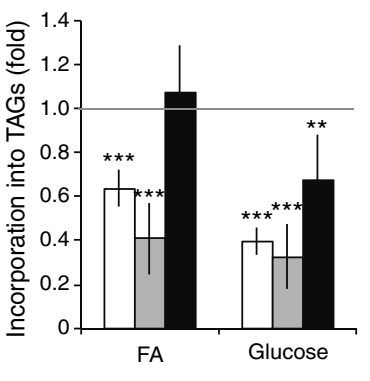

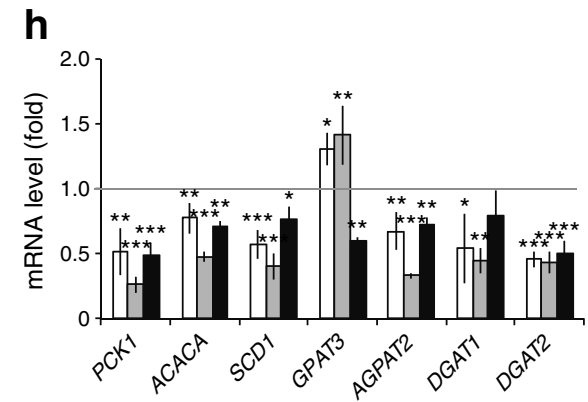

Analysis of PAP1 activity showed that lipin-1 accounted for almost all PAP1 activity (lipin-1-depleted cells showed 5\% of PAP1 activity in the control), with lipin-2 and lipin-3 single knockdowns reducing it to $49 \%$ and $61 \%$, respectively (Fig. 2e).

Next, we analysed incorporation of fatty acids and glucose into TAGs. While esterification of fatty acids was downregulated in lipin-1- and lipin-2-depleted cells, glucose incorporation into TAGs decreased in all three lipin-depleted cells (Fig. 2f).

Finally, we analysed the expression levels of transcription factors that promote early adipogenesis. $C E B P A$ was downregulated in cells depleted of any lipin family member, and $C E B P B$ was decreased upon lipin-2 deficiency (Fig. $2 \mathrm{~g}$ ). Under these conditions, gene expression of two key transcription factors regulated by CCAAT/enhancer binding proteins, PPARG and SREBPI were downregulated (Fig. 2g), and also at the protein level (ESM Fig. 2a, b). $C E B P D$ showed a significant upregulation in cells depleted of any lipin family member (Fig. $2 \mathrm{~g}$ ), probably due to the triggering of a compensation mechanism.

Given the role of lipins in neutral lipid biosynthesis, we next explored expression of lipogenic genes. The expression of these genes was significantly downregulated in cells depleted of any lipin family member (Fig. 2h). In contrast, glycerol-3-phosphate acyltransferase was significantly 
upregulated in the lipin-1 and lipin-2 knockdowns (Fig. 2h). Lipid quantification by mass spectrometry showed that, among the lipid species that were altered, the levels of most DAG (ESM Fig. 2c) and TAG (ESM Fig. 2d) species were downregulated in cells depleted of any lipin compared with controls. Interestingly, phosphatidate levels did not change (data not shown).

Overall, despite the crosstalk between lipin family members, lipin-1 silencing leads to a $95 \%$ depletion of PAP1 activity. However, our results point to a role of all three members on early stages of human adipogenesis.

\section{Single lipin silencing does not block TAG accumulation} in fully differentiated adipocytes Next, we analysed the effects of lipin silencing in SGBS pre-adipocytes on late adipogenesis. Similarly to the above experiments, we depleted each lipin member prior to induction of adipogenesis in SGBS pre-adipocytes, and cells were analysed at day 10 after differentiation (Fig. 3a), where protein downregulation of lipins still persisted (Fig. 3b and ESM Fig. 3a).

At day 10, lipin-1 depleted cells still responded by compensatory upregulation of lipin-2 protein (Fig. 3b and ESM Fig. 3a). Additionally, both single lipin-2 and lipin-3 knockdowns led to lower protein levels of the other lipins. PAP1 activity analysis showed that lipin-1 accounted for almost all PAP1 activity (lipin-1-depleted cells showed 3\% of the activity in the control). Lipin-2 and lipin-3 single knockdowns reduced PAP1 activity only slightly ( $84 \%$ and $74 \%$, respectively) (Fig. 3c).

Next, we assessed neutral lipid accumulation. Depletion of any lipin triggered a slight reduction (15\% to $34 \%)$ in TAG accumulation (Fig. 3d). Expression of key transcription factors for lipogenesis (ESM Fig. 3a, b) and genes of lipid biosynthesis (ESM Fig. 3c) were altered to a lesser extent compared with the effects observed at day 4.

Finally, fatty acid esterification into TAGs and $\alpha$-glycerophosphate synthesis/de novo lipogenesis (DNL) were downregulated in cells depleted of any lipin (Fig. 3e). Compared with day 4, lipin-3 depletion also showed reduced fatty acid esterification into TAGs at day 10 .

Overall, the effects of lipin knockdown were mild on fully differentiated adipocytes suggesting that cells overcame the initial impairment of lipid gene expression. This may be due to the existence of enough remnant protein amounts of lipins, although PAP1 activity at day 10 was still almost absent in the lipin-1 knockdowns.

Combinatorial silencing of lipins does not further alter the phenotype of fully differentiated adipocytes We then asked whether compensation by other lipin family members might help cells to recover the initial alterations shown at day 4 after adipogenesis. We used a combinatorial knockdown to deplete combinations of two of the three lipins in SGBS pre-
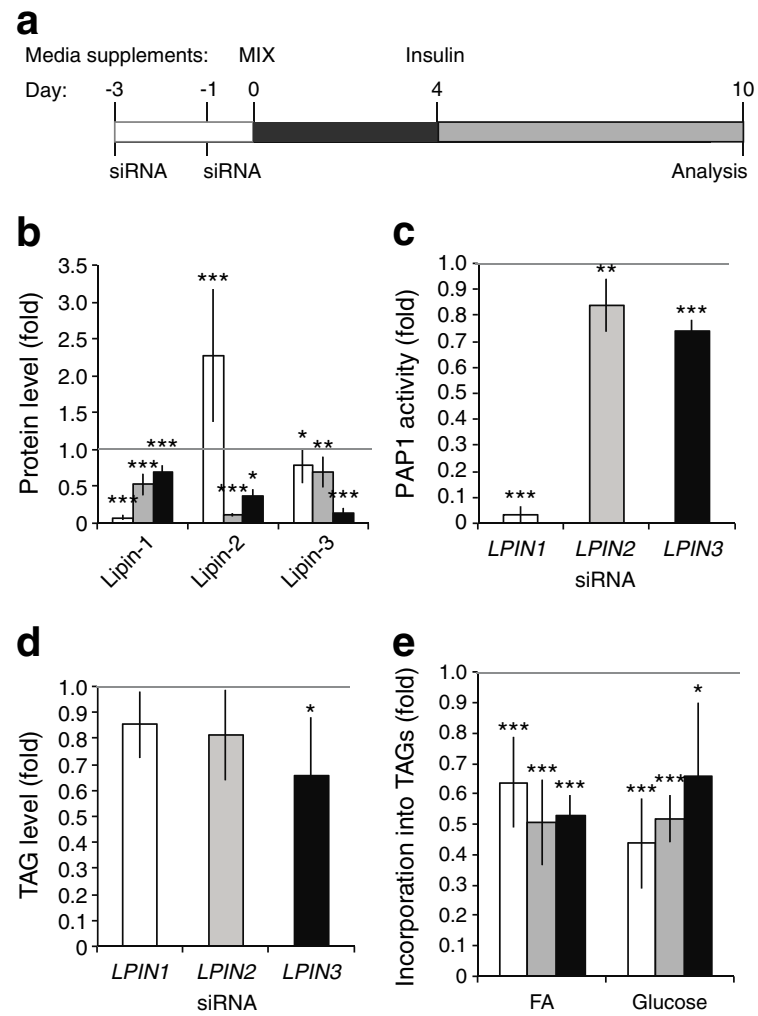

Fig. 3 Lipin-depleted SGBS pre-adipocytes can differentiate into adipocytes. (a) Knockdowns of single lipin members were performed in pre-adipocytes, adipogenesis was induced (day 0 ), and cells were collected at day 10. (b) Lipin protein levels $(n=3)$, (c) PAP1 activity (control: $\left.38.88 \pm 1.38 \mathrm{nmol} \mathrm{min}{ }^{-1} \mathrm{mg}^{-1}\right)(n=3)$, (d) total TAG content (control: $\left.110.86 \pm 37.29 \mathrm{mmol} \mathrm{l}^{-1} \mathrm{mg}^{-1}\right)(n=3)$, and (e) fatty acid (FA) and glucose incorporation into TAGs (control, fatty acids: $740.6 \pm 292.3$ arbitrary units TAG/ $\mu$ g protein, glucose: $3.73 \pm 2.60$ arbitrary units TAG/ $\mu \mathrm{g}$ protein) $(n=3)$ were analysed. Data represent mean \pm SD of fold increase over non-targeting controls (set as 1 ); $* p<0.05, * * p<0.01$, $*_{* *}<0.001$; General Lineal Model Univariate test. White bars, LPIN1 knockdown; grey bars, LPIN2 knockdown; black bars, LPIN3 knockdown

adipocytes (see Methods), and analysed cells at day 10 of differentiation (as in Fig. 3a).

Double lipin-1 and lipin-3 knockdowns led to an upregulation of lipin-2 protein levels (Fig. 4a and ESM Fig. 4). This compensatory pattern hindered the efficiency of lipin-2 knockdown in the double (lipin-1 and -2) and triple knockdown cells. Nevertheless, PAP1 activity in the triple knockdown was residual compared with the control $\left(0.18 \pm 0.31\right.$ vs $\left.39.29 \pm 3.42 \mathrm{nmol} \mathrm{min}{ }^{-1} \mathrm{mg}^{-1}\right)$.

Despite the almost complete lack of PAP1 activity, neutral lipid accumulation was not totally compromised in the triple knockdown (Fig. 4b). This is in agreement with the fact that deleterious mutations in LPIN1 do not affect adipose tissue development in humans, and it suggests that it is unlikely to be due to a compensatory upregulation of lipin-2 (Fig. 2d), since the double lipin-1 and -2 knockdown shows a similar reduction in the content of neutral lipids (Figs 3d, 4b). 


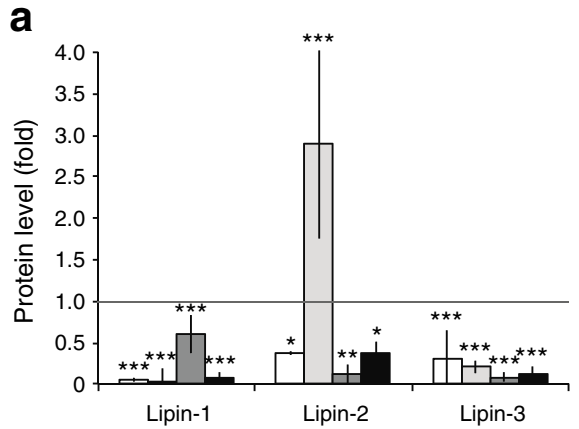

Fig. 4 Combinatorial depletion of lipins in SGBS pre-adipocytes after full differentiation. Multiple knockdowns of lipin members were performed in pre-adipocytes, adipogenesis was induced (day 0 ) and cells were collected at day 10. (a) Lipin protein levels $(n=3-6)$, (b) total TAG content (controls: $71.11 \pm 36.40$ and $101.79 \pm 76.15 \mathrm{mmol} \mathrm{l}^{-1} \mathrm{mg}^{-1}$ for double and triple knockdowns, respectively) ( $n=5)$, and (c) fatty acid (FA) and glucose incorporation into TAGs in the triple knockdown (control, fatty

Moreover, depletion of the three lipins did not significantly affect fatty acid esterification into TAGs and only slightly downregulated $\alpha$-glycerophosphate synthesis/DNL (Fig. 4c), pointing to an improvement compared with day 4 (day 4 incorporation of fatty acids: $0.52 \pm 0.35, p=0.006$, and of glucose: $0.32 \pm 0.15, p<0.001$, fold over the control level).

\section{Consumption of TAGs is reduced in lipin-depleted SGBS} adipocytes Lipid droplets accumulate neutral lipids in a dynamic manner, with a balance among lipid biosynthesis, lipid hydrolysis and FAO in mitochondria. To test whether cells protect intracellular lipids by reducing its consumption, we analysed the rates of palmitate oxidation. The results show similar FAO rates in the control, single (Fig. 5a) and triple knockdown (Fig. 5b). In contrast, levels of glycerol release were downregulated compared with controls in the single (Fig. 5c), and in all combinatorial knockdowns (Fig. 5d). Thus, reduced consumption may contribute to the accumulation of neutral lipids in lipin-depleted adipocytes.

The LPP family is upregulated under conditions of repressed lipin expression Next, we tested whether the $\mathrm{Mg}^{2+}$-independent LPPs could provide an alternative pathway for DAG synthesis. LPP activity was highly induced in the lipin-1 single knockdown (3.50 \pm 1.18 -fold increase over control levels) (Fig. 6a), and in the triple knockdown $(2.74 \pm 0.97$-fold increase) at day 10 of differentiation (Fig. 6b).

Moreover, the expression of $L P P 3$ (also known as Plpp3/ $P P A P 2 B$ ) was upregulated in the single (Fig. 6c) and in the combinatorial lipin knockdowns at day 10 (Fig. 6d) and, similarly to lipogenic genes, upregulation was higher at day four (ESM Fig. 5a). This was confirmed by using other
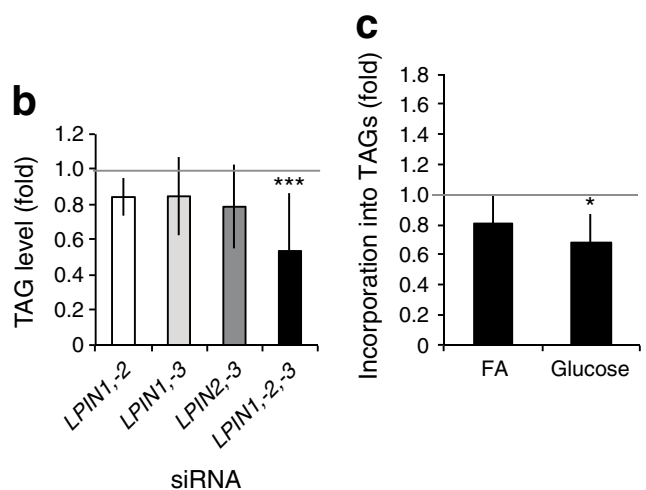

acids: $493.4 \pm 226.4$ arbitrary units TAG/ $\mu$ g protein, glucose: 3.51 \pm 2.26 arbitrary units TAG/ $\mu$ g protein $)(n=3)$ were analysed. Data represent mean $\pm \mathrm{SD}$ of fold increase over non-targeting controls (set as 1 ); ${ }^{*} p<0.05,{ }^{* *} p<0.01,{ }^{* *} p<0.001$; General Lineal Model Univariate test. White bars, LPIN1 and LPIN2 knockdown; light grey bars, LPIN1 and LPIN3 knockdown; dark grey bars, LPIN2 and LPIN3 knockdown; black bars, triple knockdown

commercial sources of lipin-1 siRNA (ESM Fig. 5b) and analysing LPP expression at day four of differentiation (ESM Fig. 5c).

Propranolol, a nonspecific $\beta$-blocker, is an effective PAP1 activity inhibitor and modestly effective as LPP inhibitor, with the LPP3 isoform being more sensitive to inhibition [36]. Propranolol treatment during adipogenesis (Fig. 6e) altered lipogenic gene expression (Fig. 6f) and blocked lipid droplet formation in SGBS adipocytes (ESM Fig. 5d).

\section{Discussion}

Herein we have addressed the contribution of the lipin paralogues to human adipogenesis. Decreased lipin expression levels and PAP1 activity in adipose tissue have been linked with insulin resistance [23-25]. In our cohort, adipose tissue expression confirmed that lipin-1 is altered in type 2 diabetes and that LPIN1 is negatively associated with insulin resistance. Moreover, we show that LPIN3 transcript levels are positively related with fasting glucose levels. This prompted us to analyse separately the effects of gene silencing of each lipin paralogue in adipogenesis.

Previous studies have established that lipin-1 plays a major role in fat metabolism in rodents, with lipin-1 deficiency causing lipodystrophy features in mice and rats $[14,38]$. The role of lipins in human adipogenesis is still undefined: mutations in the LPIN1 gene have not yet been detected in human lipodystrophy $[39,40]$. Moreover, deleterious LPIN1 mutations cause paediatric rhabdomyolysis while fat distribution, average weight and plasma biochemical variables are normal [18]. Our data suggest that the lack of an essential role for PAP1 activity in human adipogenesis is not due to 

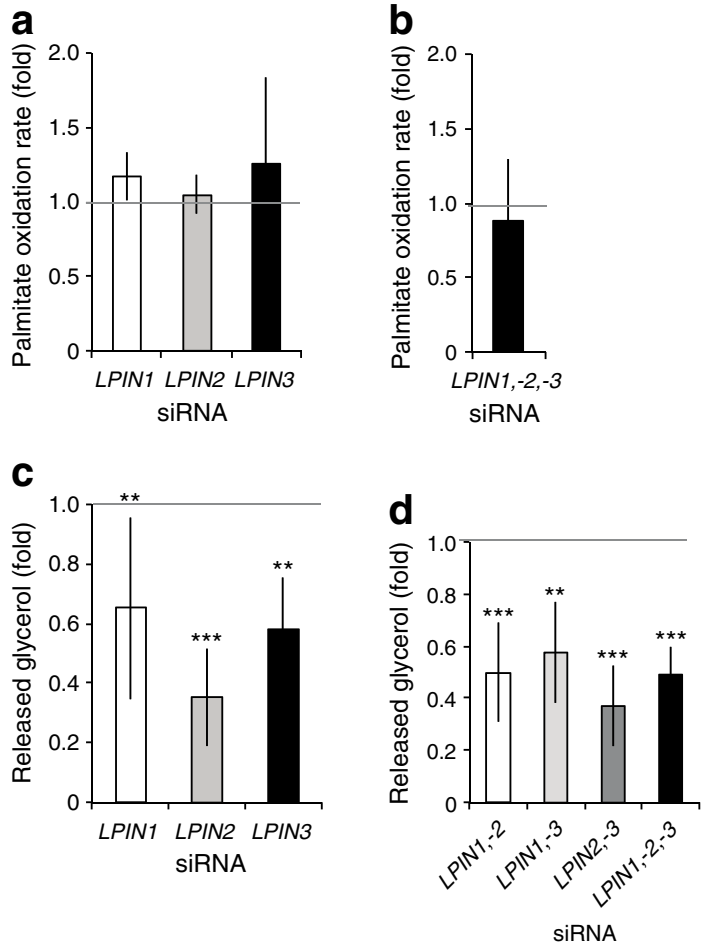

Fig. 5 Consumption of neutral lipids in fully differentiated lipin-depleted SGBS pre-adipocytes. Knockdown of single, double and triple lipin family members was performed in pre-adipocytes, adipogenesis was induced (day 0 ) and cells were collected at day 10. Palmitate oxidation rates in (a) single (control: $\left.0.77 \pm 0.37 \mathrm{nmol} \mathrm{h}^{-1} \mathrm{mg}^{-1}\right)(n=3)$, and (b) triple knockdowns (control: $\left.0.63 \pm 0.28 \mathrm{nmol} \mathrm{h}^{-1} \mathrm{mg}^{-1}\right)(n=3)$; glycerol release in (c) single (control: $\left.0.42 \pm 0.12 \mathrm{mmol}^{-1} \mathrm{mg}^{-1}\right)(n=3)$, and (d) combinatorial knockdowns (controls, double: $0.36 \pm 0.21$, and triple: $\left.0.21 \pm 0.07 \mathrm{mmol} \mathrm{l}^{-1} \mathrm{mg}^{-1}\right)(n=5)$ were analysed. Data represent mean \pm SD of fold increase over non-targeting controls (set as 1 ); ${ }^{* *} p<0.01$, $* * * p<0.001$; General Lineal Model Univariate test. (a, c) White bars, LPIN1 knockdown; grey bars, LPIN2 knockdown; black bars, LPIN3 knockdown; (b, d) white bars, LPIN1 and LPIN2 double knockdown; light grey bars, LPIN1 and LPIN3 double knockdown; dark grey bars, LPIN2 and LPIN3 double knockdown; black bars, triple knockdown

compensation by lipin-2 or lipin-3. We find that the triple lipin silencing in SGBS pre-adipocytes maintains the ability to incorporate fatty acids into TAG and accumulate neutral lipids despite loss of nearly all PAP1 activity.

SGBS pre-adipocytes are differentiated in the absence of serum and, therefore, neutral lipids should be obtained from DNL. Besides, although glyceroneogenesis is the major pathway for glycerol synthesis in the mature adipocyte [41], during adipogenesis glycolysis may account for an important source of $\alpha$-glycerophosphate. Thus, glucose incorporation into TAGs may account for DNL, but also for $\alpha$-glycerophosphate synthesis via glyceroneogenesis and glycolysis. Downregulated $\alpha$-glycerophosphate synthesis/ DNL points to broader effects of lipin silencing other than TAG synthesis. Moreover, the putative intranuclear roles of lipin-1, -2 and -3 in SGBS adipocytes add some complexity to the above.

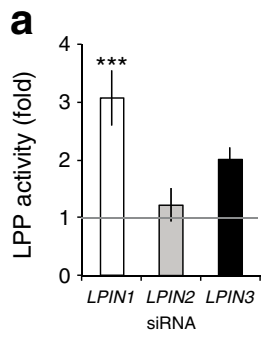

b
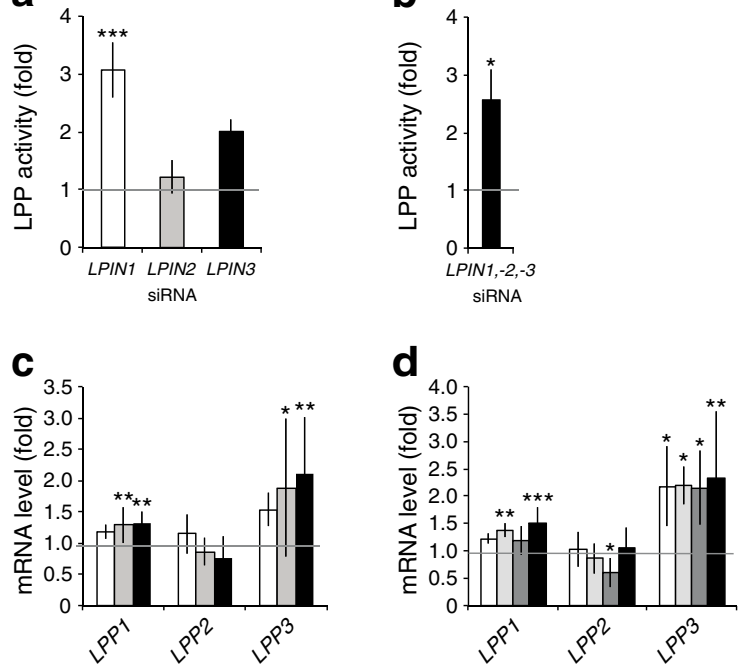

e

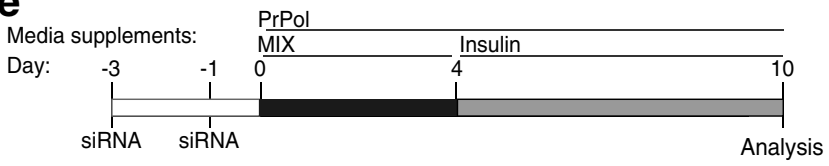

f

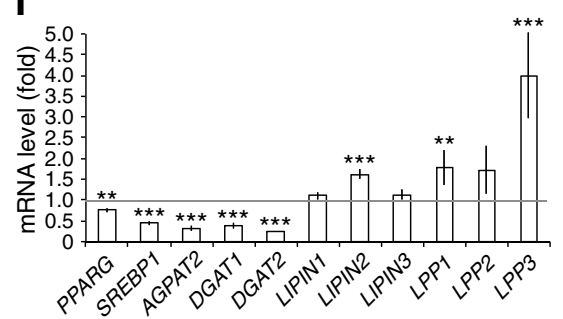

Fig. 6 Induction of the LPP family under conditions of repressed lipin expression. Knockdowns of single, double and triple lipin family members were performed in pre-adipocytes, adipogenesis was induced (day 0) and cells were collected at day 10. LPP activity in (a) the single (control: $\left.6.01 \pm 0.60 \mathrm{nmol} \mathrm{min}^{-1} \mathrm{mg}^{-1}\right)(n=3)$ and (b) triple knockdowns (control: $\left.4.82 \pm 0.53 \mathrm{nmol} \mathrm{min}{ }^{-1} \mathrm{mg}^{-1}\right)(n=3)$, and expression levels of the LPP family in (c) the single $(n=7)$ and (d) combinatorial knockdowns $(n=4-7)$ were analysed. (e) SGBS pre-adipocytes were induced to differentiate in presence of $100 \mu \mathrm{mol} / 1$ of propranolol (PrPol). (f) mRNA levels of lipogenic genes were analysed $(n=3)$. Data represent mean \pm SD of fold increase over non-targeting controls $(\mathbf{a}-\mathbf{d})$ or non-treated control (f); ${ }^{*} p<0.05,{ }^{* *} p<0.01, * * * p<0.001$; General Lineal Model Univariate test. (a, c) White bars, LPIN1 knockdown; grey bars, LPIN2 knockdown; black bars, LPIN3 knockdown; (b, d) white bars, LPIN1 and LPIN2 double knockdown; light grey bars, LPIN1 and LPIN3 double knockdown; dark grey bars, LPIN2 and LPIN3 double knockdown; black bars, triple knockdown; (f) white bars, propranolol-treated cells

The lipin family members cooperate for optimal PAP activity in mouse adipose tissue [42], liver [43, 44], brain [43] and human primary myoblasts [45]. Our data show that human lipin-1 accounts for most PAP1 activity in SGBS adipocytes and that upregulation of lipin-2 in lipin-1depleted cells does not compensate for PAP1 activity. By contrast, downregulation of PAP1 activity in lipin-2 and lipin-3 single knockdowns cannot be fully attributed to their own depletion since they also downregulate lipin-1. 
Activation of non-lipin compensatory pathways may also mask the lipin phenotypes. Nevertheless, (1) we found no evidence, at the mRNA level, of upregulation of the monoacylglycerol $O$-acyltransferase pathway that can generate DAG from monoacylglycerol [46, 47] (data not shown); and (2) cholesteryl esters levels are likely not upregulated since the effect of lipin knockdown on neutral lipid content looks similar by Oil Red O staining (ORO) and TAG content (ORO: $0.76 \pm 0.06$, and TAG: $0.85 \pm 0.13$ in the lipin-1 knockdown, and ORO: $0.74 \pm 0.12$, and TAG: $0.54 \pm 0.33$ in the triple lipin knockdown, compared with the control set as 1). In contrast, LPP activity was induced in the lipin knockdowns, possibly due to an earlier upregulation of their transcript levels (observed by day 4). Moreover, inhibition of both lipins and LPPs with propranolol completely blocked lipid droplet formation, although this fact must be taken with caution because of the non-specificity of this compound. LPPs hydrolyse phosphatidate as well as different substrates, and act on the outer surface of plasma membrane and in the luminal surface of endoplasmic reticulum (ER) and Golgi membranes [6] and thus, it is not clear if they can have access to the phosphatidate formed from glycerol phosphate and acyl-CoA. However, in yeast, TAG synthesised both in the cytosolic and luminal leaflets of the ER membranes are efficiently packed into lipid droplets [48].

Another strategy to protect the lipid storage when glycerolipid synthesis is compromised might be downregulation of lipid hydrolysis and FAO. Lipin-1 regulates basal lipolysis [49], and modulates FAO transcript expression levels [9]. We show that all combinatorial knockdowns of lipins downregulate basal lipolysis to a greater extent than reduction of neutral lipid content.

In summary, we confirm that adipose tissue expression of the lipin family is altered in type 2 diabetes. Furthermore, loss of nearly all PAP1 activity, due to combined lipin-1, -2 and -3 knockdown, has only mild effects on final adipogenesis and lipid accumulation in SGBS cells. Conversely, lipin-2 and lipin-3 may contribute little to total PAP activity, but still play a role in early adipogenesis. Our results suggest a compensation strategy to accumulate near normal neutral lipid levels, activating other pathways (such as LPPs) and inhibiting TAG hydrolysis. More work is clearly required to decipher whether LPPs or other unknown pathways may compensate lack of PAP1 activity.

Acknowledgements We are grateful to I. Mylonis (Laboratory of Biochemistry, Faculty of Medicine, University of Thessaly, Greece) for advice with the cell fractionation protocol.

Funding This study was supported by research grants from the 'Instituto de Salud Carlos III' (ISCIII, Spanish Ministry of Economy and Competitiveness) (PI10/00967 and CP11/00021 to MM); the R. Barri Private Foundation (PV12142S to MM); the Medical Research Council (G0701446 to SS); and National Institutes of Health Grant
(GM028140 to GMC). CIBER de Diabetes y Enfermedades Metabólicas asociadas (CB07708/0012) is an initiative of the ISCIII. MM acknowledges support from the 'Miguel Servet' tenure track programme (CP11/00021), from the Fondo de Investigación Sanitaria (FIS) co-financed by the European Regional Development Fund (ERDF), and supported by a Salvador de Madariaga Mobility fellowship from the Spanish Ministry of Education (PR2011-0584). AT is the recipient of a FI-DGR fellowship (9015-97318/2012) from the Agència de Gestió d'Ajuts Universitaris i de Recerca (AGAUR). The funders had no role in study design, data collection and analysis, decision to publish, or preparation of the manuscript.

Duality of interest The authors declare that there is no duality of interest associated with this manuscript.

Author contributions SS, AZ and MM were the main contributors in the conception, design and interpretation of the data, and in writing the manuscript. AT, HS, G-SH, DS, JC, CM and MM performed the experiments and data analysis, and revised the manuscript. JG, MW, $\mathrm{CR}, \mathrm{OY}$ and GMC were responsible for acquisition of data and analysis, and revised the manuscript. All authors had final approval of the submitted and published versions. MM is the guarantor of this work and, as such, had full access to all the data in the study and takes responsibility for the integrity of the data and the accuracy of the data analysis.

Open Access This article is distributed under the terms of the Creative Commons Attribution 4.0 International License (http:// creativecommons.org/licenses/by/4.0/), which permits unrestricted use, distribution, and reproduction in any medium, provided you give appropriate credit to the original author(s) and the source, provide a link to the Creative Commons license, and indicate if changes were made.

\section{References}

1. Athenstaedt K, Daum G (2006) The life cycle of neutral lipids: synthesis, storage and degradation. Cell Mol Life Sci 63: $1355-1369$

2. Unger RH (2002) Lipotoxic diseases. Annu Rev Med 53:319-336

3. Huang-Doran I, Sleigh A, Rochford JJ, O'Rahilly S, Savage DB (2010) Lipodystrophy: metabolic insights from a rare disorder. J Endocrinol 207:245-255

4. Han GS, Wu WI, Carman GM (2006) The Saccharomyces cerevisiae lipin homolog is a $\mathrm{Mg}^{2+}$-dependent phosphatidate phosphatase enzyme. J Biol Chem 281:9210-9218

5. Donkor J, Sariahmetoglu M, Dewald J, Brindley DN, Reue K (2007) Three mammalian lipins act as phosphatidate phosphatases with distinct tissue expression patterns. J Biol Chem 282: 3450-3457

6. Brindley DN, Pilquil C, Sariahmetoglu M, Reue K (2009) Phosphatidate degradation: phosphatidate phosphatases (lipins) and lipid phosphate phosphatases. Biochim Biophys Acta 1791: 956-961

7. Harris TE, Finck BN (2011) Dual function lipin proteins and glycerolipid metabolism. Trends Endocrinol Metab 22:226-233

8. Donkor J, Zhang P, Wong S et al (2009) A conserved serine residue is required for the phosphatidate phosphatase activity but not the transcriptional coactivator functions of lipin-1 and lipin-2. J Biol Chem 284:29968-29978 
9. Finck BN, Gropler MC, Chen $\mathrm{Z}$ et al (2006) Lipin 1 is an inducible amplifier of the hepatic PGC-1alpha/PPARalpha regulatory pathway. Cell Metab 4:199-210

10. Koh YK, Lee MY, Kim JW et al (2008) Lipin1 is a key factor for the maturation and maintenance of adipocytes in the regulatory network with CCAAT/enhancer-binding protein alpha and peroxisome proliferator-activated receptor gamma 2. J Biol Chem 283: 34896-34906

11. Liu GH, Gerace L (2009) Sumoylation regulates nuclear localization of lipin-1alpha in neuronal cells. PLoS One 4, e7031

12. Peterson TR, Sengupta SS, Harris TE et al (2011) mTOR complex 1 regulates lipin 1 localization to control the SREBP pathway. Cell 146:408-420

13. Kim HB, Kumar A, Wang L et al (2010) Lipin 1 represses NFATc4 transcriptional activity in adipocytes to inhibit secretion of inflammatory factors. Mol Cell Biol 30:3126-3139

14. Péterfy M, Phan J, Xu P, Reue K (2001) Lipodystrophy in the fld mouse results from mutation of a new gene encoding a nuclear protein, lipin. Nat Genet 27:121-124

15. Nadra K, Médard JJ, Mul JD et al (2012) Cell autonomous lipin 1 function is essential for development and maintenance of white and brown adipose tissue. Mol Cell Biol 32:4794-4810

16. Zhang P, Takeuchi K, Csaki LS, Reue K (2012) Lipin-1 phosphatidic phosphatase activity modulates phosphatidate levels to promote peroxisome proliferator-activated receptor $\gamma(\operatorname{PPAR} \gamma)$ gene expression during adipogenesis. J Biol Chem 287:3485-3494

17. Grimsey N, Han GS, O'Hara L, Rochford JJ, Carman GM, Siniossoglou S (2008) Temporal and spatial regulation of the phosphatidate phosphatases lipin 1 and 2. J Biol Chem 283: 29166-29174

18. Zeharia A, Shaag A, Houtkooper RH et al (2008) Mutations in LPIN1 cause recurrent acute myoglobinuria in childhood. Am J Hum Genet 83:489-494

19. Zhang R, Jiang F, Hu C et al (2013) Genetic variants of LPIN1 indicate an association with type 2 diabetes mellitus in a Chinese population. Diabet Med 30:118-122

20. Bego T, Dujic T, Mlinar B et al (2011) Association of PPARG and LPIN1 gene polymorphisms with metabolic syndrome and type 2 diabetes. Med Glas (Zenica) 8:76-83

21. Aulchenko YS, Pullen J, Kloosterman WP et al (2007) LPIN2 is associated with type 2 diabetes, glucose metabolism, and body composition. Diabetes 56:3020-3026

22. Loos RJ, Rankinen T, Pérusse L, Tremblay A, Després JP, Bouchard C (2007) Association of lipin 1 gene polymorphisms with measures of energy and glucose metabolism. Obesity 15:2723-2732

23. Suviolahti E, Reue K, Cantor RM et al (2006) Cross-species analyses implicate Lipin 1 involvement in human glucose metabolism. Hum Mol Genet 15:377-386

24. Yao-Borengasser A, Rasouli N, Varma V et al (2006) Lipin expression is attenuated in adipose tissue of insulin-resistant human subjects and increases with peroxisome proliferator-activated receptor gamma activation. Diabetes 55:2811-2818

25. Saggerson ED (1988) Phosphatidate phosphohydrolase activity in adipose tissue. In: Brindley DN (ed) Phosphatidate phosphohydrolase. Volume I. CRC Press, Boca Raton, pp 79-124

26. Miranda M, Escoté X, Ceperuelo-Mallafré V et al (2010) Paired subcutaneous and visceral adipose tissue aquaporin-7 expression in human obesity and type 2 diabetes: differences and similarities between depots. J Clin Endocrinol Metab 95:3470-3479

27. Miranda M, Escoté X, Alcaide MJ et al (2010) Lpin1 in human visceral and subcutaneous adipose tissue: similar levels but different associations with lipogenic and lipolytic genes. Am J Physiol Endocrinol Metab 299:E308-E317

28. World Health Organization (2000) Obesity: preventing and managing the global epidemic. Report of a WHO Consultation (WHO Technical Report Series 894). WHO, Geneva
29. International Diabetes Federation (2006) IDF consensus worldwide definition of the metabolic syndrome. Available from www.idf.org/ webdata/docs/MetS_def update2006.pdf, accessed 24 Nov 2015

30. Wabitsch M, Brenner RE, Melzner I et al (2001) Characterization of a human preadipocyte cell strain with high capacity for adipose differentiation. Int J Obes Relat Metab Disord 25:8-15

31. Fischer-Posovszky P, Newell FS, Wabitsch M, Tornqvist HE (2008) Human SGBS cells - a unique tool for studies of human fat cell biology. Obes Facts 1:184-189

32. Sembongi H, Miranda M, Han GS et al (2013) Distinct roles of the phosphatidate phosphatases lipin 1 and 2 during adipogenesis and lipid droplet biogenesis in 3T3-L1 cells. J Biol Chem 288: 34502-34513

33. Sebastián D, Guitart M, García-Martínez C et al (2009) Novel role of FATP1 in mitochondrial fatty acid oxidation in skeletal muscle cells. J Lipid Res 50:1789-1799

34. Bradford MM (1976) A rapid and sensitive method for the quantitation of microgram quantities of protein utilizing the principle of protein-dye binding. Anal Biochem 72:248-254

35. Carman GM, Lin YP (1991) Phosphatidate phosphatase from yeast. Methods Enzymol 197:548-553

36. Han GS, Carman GM (2004) Assaying lipid phosphate phosphatase activities. Methods Mol Biol 284:209-216

37. Han GS, Carman GM (2010) Characterization of the human LPIN1-encoded phosphatidate phosphatase isoforms. J Biol Chem 285:14628-14638

38. Mul JD, Nadra K, Jagalur NB et al (2011) A hypomorphic mutation in Lpin 1 induces progressively improving neuropathy and lipodystrophy in the rat. J Biol Chem 286:26781-26793

39. Cao H, Hegele RA (2002) Identification of single-nucleotide polymorphisms in the human LPIN1 gene. J Hum Genet 47:370-372

40. Fawcett KA, Grimsey N, Loos RJ et al (2008) Evaluating the role of LPIN1 variation in insulin resistance, body weight, and human lipodystrophy in U.K. populations. Diabetes 57:2527-2533

41. Nye CK, Hanson RW, Kalhan SC (2008) Glyceroneogenesis is the dominant pathway for triglyceride glycerol synthesis in vivo in the rat. J Biol Chem 283:27565-27574

42. Csaki LS, Dwyer JR, Li X et al (2013) Lipin-1 and lipin-3 together determine adiposity in vivo. Mol Metab 3:145-154

43. Dwyer JR, Donkor J, Zhang P et al (2012) Mouse lipin-1 and lipin-2 cooperate to maintain glycerolipid homeostasis in liver and aging cerebellum. Proc Natl Acad Sci U S A 109:E2486-E2495

44. Schweitzer GG, Chen Z, Gan C et al (2015) Liver-specific loss of lipin-1-mediated phosphatidic acid phosphatase activity does not mitigate intrahepatic TG accumulation in mice. J Lipid Res 56: 848-858

45. Michot C, Mamoune A, Vamecq J et al (2013) Combination of lipid metabolism alterations and their sensitivity to inflammatory cytokines in human lipin-1-deficient myoblasts. Biochim Biophys Acta 1832:2103-2114

46. Banh T, Nelson DW, Gao Y, Huang TN, Yen MI, Yen CL (2015) Adult-onset deficiency of acyl CoA:monoacylglycerol acyltransferase 2 protects mice from diet-induced obesity and glucose intolerance. J Lipid Res 56:379-389

47. Shi Y, Cheng D (2009) Beyond triglyceride synthesis: the dynamic functional roles of MGAT and DGAT enzymes in energy metabolism. Am J Physiol Endocrinol Metab 297:E10-E18

48. Choudhary V, Jacquier N, Schneiter R (2011) The topology of the triacylglycerol synthesizing enzyme Lro1 indicates that neutral lipids can be produced within the luminal compartment of the endoplasmatic reticulum: implications for the biogenesis of lipid droplets. Commun Integr Biol 4:781-784

49. Mitra MS, Chen Z, Ren $\mathrm{H}$ et al (2013) Mice with an adipocytespecific lipin 1 separation-of-function allele reveal unexpected roles for phosphatidic acid in metabolic regulation. Proc Natl Acad Sci U S A 110:642-647 